\title{
La nacionalidad de las otras comunicaciones
}

Eduardo Barrera Herrera

El Colegio de la Frontera Norte

Robert REICH (1992), Secretario del Trabajo y principal asesor económico de la administración Clinton, asegura que están desapareciendo las compañías y los productos norteamericanos (o franceses, japoneses, alemanes, etc.) en virtud de las redes globales de producción que hacen obsoletas las concepciones de las economías como nacionales y en las que los proteccionismos comerciales son peligrosos atavismos. Por otra parte, $\mathrm{Al} \mathrm{Gore,} \mathrm{vicepresidente} \mathrm{de} \mathrm{dicha} \mathrm{administra-}$ ción, es el principal promotor de la "carretera electrónica" que se convertiría en la columna vertebral de los esfuerzos orquestados de diversos sectores (militar, educativo y privado) en investigación y desarrollo de los que se obtendría como resultado final un subsidio al último sector mediante la transferencia de tecnología. Ambos procesos aparentemente aislados o que sugieren cierta contradicción revelan tres aspectos claves de la nueva economía global: (a) un discurso económicamente liberal que (b) oculta mecanismos para proteger y estimular las corporaciones que compiten en los mercados globales, que (c) tienen como componente clave la infraestructura de telecomunicaciones.

Aunque Reich acepta la existencia de cierta división del trabajo en las redes globales y de manera paternalista la llega a considerar como una ayuda al Tercer Mundo, ignora las grandes asimetrías no sólo entre las naciones, sino entre el capital que no reconoce fronteras y los mercados laborales nacionales por los que fluye espacial e informáticamente.

El discurso no pudo haber encontrado mejor contraparte que gobiernos como el mexicano, el cual adopta como propio el elemento retórico sin reparar en las nuevas relaciones entre los capitales multinacionales y los estados nacionales. 
Ante este discurso, los medios académicos latinoamericanos se muestran sin capacidad para el análisis crítico efectivo no sólo por el surgimiento de nuevos objetos de estudio (lo popular, procesos de recepción, etc.), sino por el desgaste y la obsolesencia de perspectivas teóricas como el imperialismo cultural y dependencia cultural. Además de los errores teóricos y epistemológicos de dichos enfoques, el propio contexto material es irreconocible para quienes afirmaban que "los medios eran americanos". Aunque Schiller aún afirma que "la preeminencia mundial de la esfera de medios / cultural norteamericana permanece intacta, si no es que más segura que nunca" (1992: 1) basándose en el soporte ideológico de los medios a eventos como la Guerra del Golfo, es un hecho que las industrias culturales han cambiado radicalmente en cuanto a la estructura de los mercados mundiales y la propiedad. El mercado mundial de la televisión se ha vuelto más complejo y su descripción como flujo unidireccional ha tenido que matizarse debido a los crecientes intercambios regionales y las exportaciones de compañías como Rede Globoy Televisa (Varis, 1984). Solamente unade las cincograndes casas grabadoras es norteamericana (Rutten, 1991) y seis de las "siete grandes" del cine han sido adquiridas gradualmente por capitales japoneses, australianos, italianos y británi$\cos$ (Smith, 1991). La existencia de un "imperio americano" (Schiller, 1992) sustentada en el dominio de sus industrias culturales en el mercado mundial parece haberse esfumado. Dicho esquema concebía a las industrias culturales transnacionales como tentáculos del estado-nación industrializado, subsumiendo la lógica económica a un nacionalismo esencialista. En el caso de los estados latinoamericanos, eran normativamente concebidos como situados por encima del interés de clase y en la práctica instrumentos de las corporaciones domésticas de importancia internacional aliadas a las potencias extranjeras.

La dominante presencia de compañías norteamericanas se encuentra lejos de esfumarse en las otras industrias de comunicación: las telecomunicaciones. A diferencia de los mecanismos nacionales para perpetuar el control de los mercados, como se hizo por décadas en la industria cinematográfica a través de la Motion Picture Export Association of America, las firmas de telecomunicaciones buscan cartelizarse por medio de alianzas estratégicas en las que resulta cada vez más irrelevante la nacionalidad de sus socios. La nueva economía global multipolar pareciera revertir la antigua relación instrumentalista y presentarnos estados nacionales que son acompañados de organizaciones intergubernamentales (Banco Mundial, Fondo Monetario Internacional, GATT, Unión Internacional de Teleco- 
municaciones, etc.) en la hipóstasis de la lógica del capital una vez que se han diluido las estructuras corporativas y los compromisos de bienestar.

\section{Las telecomunicaciones como mercancía}

Las telecomunicaciones han sido descritas como la infraestructura clave en la llamada era de la información. La concepción de esta infraestructura como factor de desarrollo económico y social ha persistido en el uso de analogías con la infraestructura de transportación. El término de "carretera electrónica" describe un proyecto de redes de servicios integrados para impulsar la investigación, desarrollo y transferencia de tecnología. Dicha etiqueta no es nueva, habiendo sido utlizada por un periodista estadounidense hace casi un cuarto de siglo al especular sobre los usos potenciales de la televisión por cable con capacidad interactiva (Smith, 1970). A pesar de la continuidad en la nomenclatura, hay enormes diferencias en las premisas y concepciones más de dos décadas después. La "carretera electrónica" de Smith era concebida como un bien público que suponía la aplicación del principio de acceso universal que rige al servicio telefónico en ese país. Esta idea original preveía una sociedad donde se utilizarían las nuevas vías bidereccionales para sufragar, estudiar, vender, comprar y realizar otras transacciones desde el hogar. La actual concepción ha abandonado la idea de las telecomunicaciones como servicio público y puede seguir utilizando la misma etiqueta con la diferencia de que ahora se trata de una autopista de cuota a la que hay un acceso desigual por parte de los diferentes estratos socioeconómicos.

La mercantilización de las telecomunicaciones en México ha traído como consecuencia la privatización y transnacionalización de la infraestructura, lo que afecta negativamente a los pequeños usuarios y a los demandantes de servicios básicos.

\section{La transnacionalización de los satélites}

El primer signo de la tendencia a estructurar mercados de servicios fue la comunicación satelital. El Sistema Morelos consistía en dos satélites híbridos (bandas C y Ku) lanzados en 1985 por el gobierno federal después de haber enmendado la constitución para reservar al Estado dicha actividad y apropiarse del proyecto satelital de Televisa. La inversión de 220 millones de dólares de un país endeudado 
fue justificada aludiendo la implementación de proyectos de telefonía rural, telemedicina, educación a distancia, etc. Actualmente, los dos satélites están saturados primordialmente por señales de televisión y datos, después de que durante el primer tercio de su vida útil eran tremendamente subutilizados, ocupándose sólo una cuarta parte del sistema. De las aplicaciones mencionadas al justificar la inversión hubo sólo esporádicas videoconferencias entre hospitales infantiles.

Aunque el Artículo 28 constitucional reserva para el Estado la explotación de los satélites Telecom, la empresa paraestatal que opera la comunicación satelital ha firmado convenios con varias empresas estadounidenses para la comunicación transfronteriza. Dichas compañías son:

1. Telepuerto Internacional de Houston. A través de STARS, este telepuerto baja la señal en sus propias instalaciones y tiene un solo cliente importante.

2. Overseas Telecommunications Incorporated (OTI). OTI tiene 19 usuarios transfronterizos, 5 en el IBS de Intelsat con enlaces en los estados estadounidenses de Arizona, Michigan, Florida y Maryland. En el Sistema Morelos tiene 14 clientes que tienen como destino estadounidense el Telepuerto Internacional Dallas-Fort Worth (Figura 1).

3. Vitacom. Esta compañía con base en Mountain View maneja un pequeño mercado de plantas manufactureras donde no existe otra infraestructura.

En 1994 serán lanzados los satélites Solidaridad, los cuales han sido diseñados y planeados con una orientación abiertamente comercial. Telecom, la empresa paraestatal que opera la comunicación satelital, tiene ya garantizada la demanda para los 14 años de vida útil de dicho sistema por parte de usuarios a lo largo del continente, incluyendo a Chile. 
Figura 1. Red de Overseas Telecommunications Incorporated (OTI)

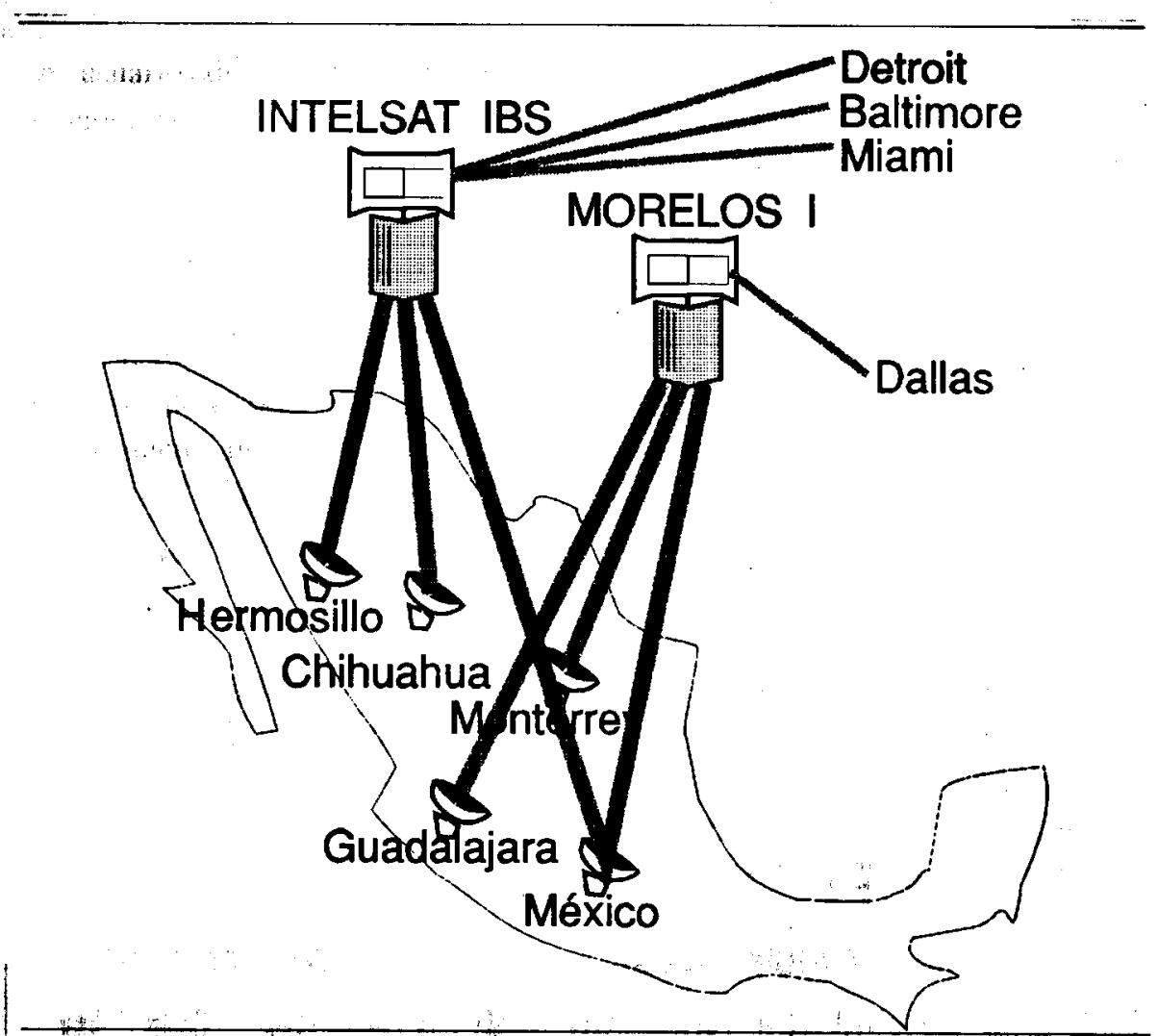

Inversión extranjera en telefonía celular

La segunda área del sector telecomunicaciones en seguir una orientación eminentemente comercial fue la telefonía celular caracterizada por la falta de regulación y cuyo concurso resultó en más de 140 ofertas a la Secretaría de Comunicaciones y Transportes. La asignación de concesiones se llevó a cabo tras dividir el territorio nacional en nueve regiones, reservando una banda a Telmex a través de Dipsa, una de sus subsidiarias. Dentro de los inversionistas nacionales figuran poderosos grupos como IUSA, TAMSA y Protexa. Por parte de los inversionistas extranjeros destacan Bell Canada, Motorola, Mitsubishi y Bell South (Figura 2). 
Figura 2. Inversión extranjera en telefonía celular

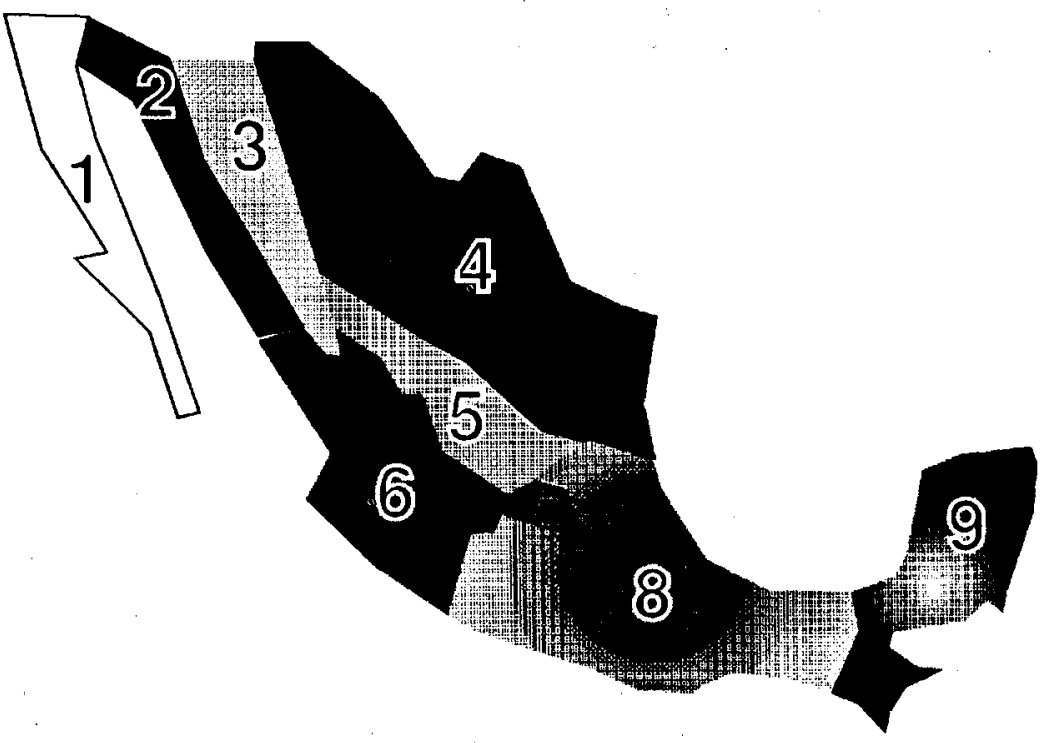

\begin{tabular}{|c|c|}
\hline COMPAŃ IA & SOCIO EXTRANJERO \\
\hline $\begin{array}{l}1\llcorner\text { Baja Celular México } \\
2 \square \text { Movitel del Noroeste }\end{array}$ & $\begin{array}{l}\text { General Cellular Corp. } \\
\text { Nc. Caw Cellular Comm. Contel Celluar }\end{array}$ \\
\hline 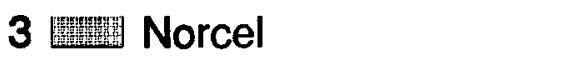 & Motorola \\
\hline Celtel & Millicom \\
\hline 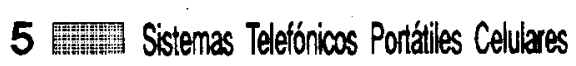 & Bell Canada \\
\hline - Comunicaciones Celutares de Occidente & Bell South \\
\hline 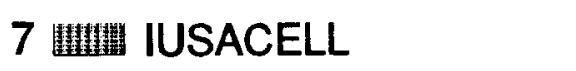 & Mitsubishi \\
\hline 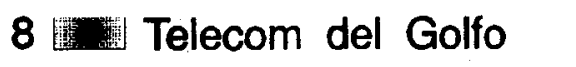 & Bell Canada \\
\hline 9 Portatel del Sureste & ND \\
\hline
\end{tabular}




\section{Las nuevas políticas tarifarias}

Los usuarios del servicio telefónico básico han sufrido el abandono de una concepción de éste como servicio público. Las tarifas telefónicas han sufrido incrementos sin precedentes en lo que se refiere al servicio medido y la larga distancia nacional, mientras que la larga distancia internacional ha tenido reducciones (Figura 3). Este cambio afecta negativamente no sólo a los usuarios de bajos ingresos que tienen que destinar una porción significativamente mayor del presupuesto familiar en términos relativos y absolutos, sino que descalifica a un gran segmento de la población que demandaba dicho servicio y al que le resultaría prácticamente imposible pagarlo.

Figura 3. Tarifas de Telmex por servicio

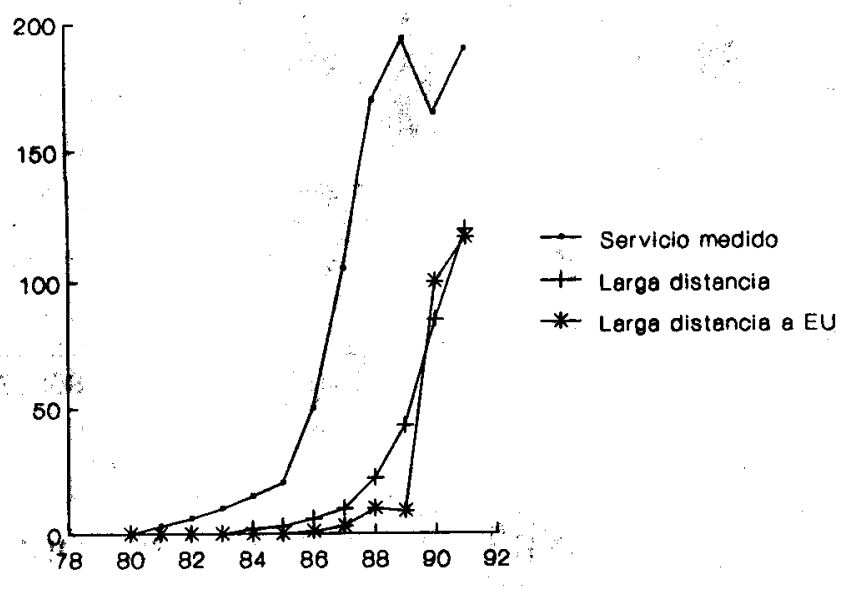

Los cambios obedecen a la nueva política tarifaria basada en costos que sustitutye a la antigua política tarifaria basada en demanda. Las nuevas tarifas recaen en la parte más cara del sistema (red local), liberando a los usuarios de larga distancia internacional, cuya demanda había crecido a una tasa anual del $50 \%$, que es cinco veces superior a la de la larga distancia nacional (Figura 4). Esta transformación 
es parte de un fenómeno más amplio que se cristaliza en la reprivatización de Teléfonos de México en 1990. Un análisis más detallado de las circunstancias que rodearon la venta de una de las compañías revela los factores endógenos y exógenos que han moldeado el sector de telecomunicaciones en México.

Figura 4. Tráfico telefónico en México 1985-1989 (106)

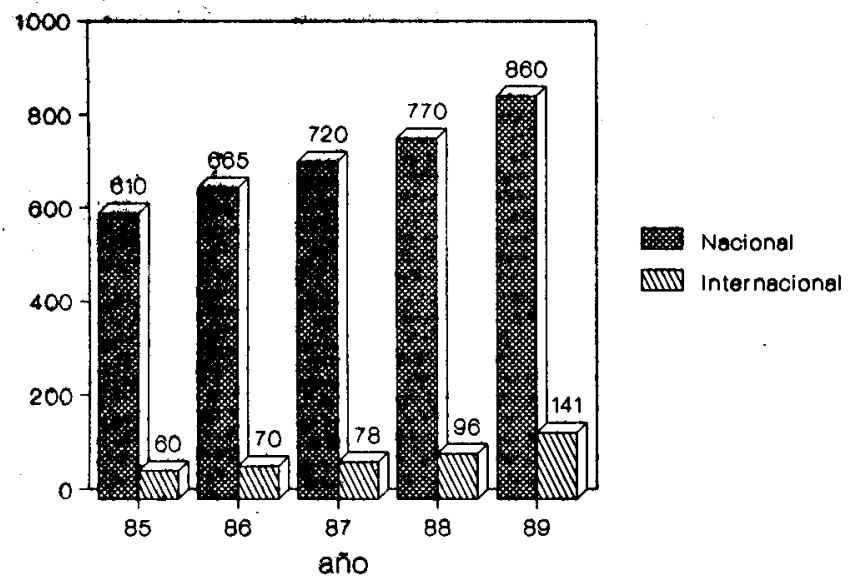

Fuente de datos: Teléfonos de México, S.A. de C.V. (1990: 12-13).

Del endeudamiento a la desnacionalización: la reprivatización de Telmex

Telmex no es ni la primera ni la última compañía telefónica que se ha privatizado en América Latina. Chile fue el primer país de la región en llevar a cabo la desincorporación de Entel y crC en 1988. México y Argentina le siguieron a finales de 1990 y Venezuela en 1991. Otros países de la región que privatizaron el servicio telefónico incluyen a Jamaica, Bermudas, San Kitts y Belice. Uruguay, Perú y Costa Rica han ya iniciado dicho proceso. 
Los análisis de las políticas nacionales de telecomunicaciones y el rol de ganismos internacionales tienden a plantear la privatización de las telecomuniciones aislando el sector del contexto económico general y como un fenómeno minentemente ideológico (Mosco, 1989; Samarajiva, 1990 y Hills, en prensa). jjo esta concepción, el neoliberalismo económico y el neoconservadurismo sítico serían exportados por las economías centrales y las organizaciones interıcionales dominadas por éstos hacia el tercer mundo, resultando en la ercantilización, privatización y desregulación de las telecomunicaciones. El nómeno de desincorporación no es exclusivo del sector telecomunicaciones. En 191 subsistían en México sólo el 18\% de las 1,155 empresas paraestatales que istían en 1982 (Figura 5).

Figura 5. Desincorporación de empresas paraestatales en México

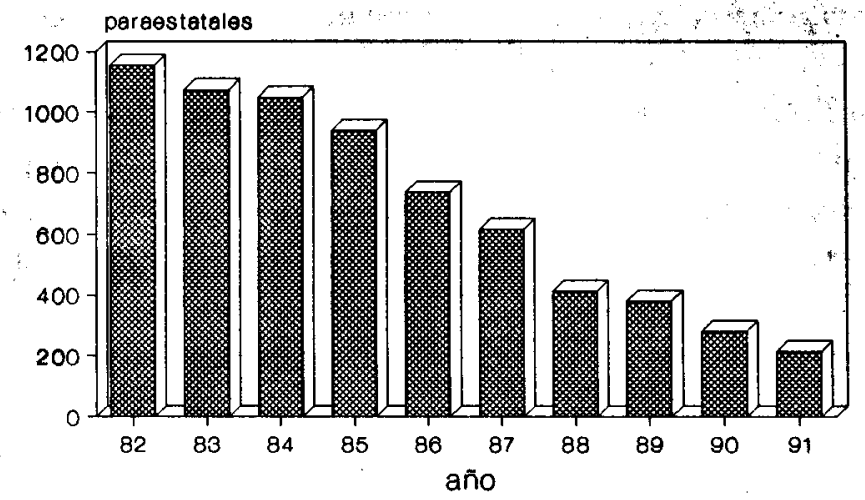

Fuente de datos: Tandon (1992).

or otro lado, la aplicación del neoliberalismo económico en la región no ha sido ślo producto de la exportación de una ideología, aunque se haya dado una difusión rquestada por organizaciones como el Banco Mundial, el FMI y la USAID. Aunque 
dichas políticas anteceden al abierto activismo en el seno de dichos organismos, 1 su difusión es efectiva sólo cuando se presentan las condiciones materiales apropiadas. Estas condiciones aparecieron en la crisis de deuda externa de América Latina quéestalló en $1982 .{ }^{2}$ La deuda externa de América Latina es generalmente aceptada como válida en círculos académicos y oficiales y se debate solamente acerca de las condiciones y términos del pago de la misma. Concepciones alternativas de la misma ven sus orígenes en la crisis petrolera de los años setenta al establecerse mecanismos para transferir valor de los consumidores occidentales a los capitales occidentales vía petrodólores. Estos se invirtieron en actividades especulativas y se disminuyeron las importanciones no petroleras creando un déficit comercial en América Latina. Esto dio lugar a que el FMI iniciara la batalla contra las "rigideces estructurales" como sindicalismo e indexación de salarios. Los países deudores tuvieron que renegociar, siempre con la previa aprobación del FMI que imponía severas reducciones en el gasto público, eliminación de subsidios, "desnacionalización y privatización para romper la cuña de los trabajadores con el Estado" (Cleaver, 1990: 31).

En 1985 el gobierno estadounidense presentó el Plan Baker en una reunión del FMI. Este constituía el primer esfuerzo por tratar de manera integral la deuda externa del Tercer Mundo. Diez de los 18 países involucrados eran latinoamericanos y deberían abrir sus economías a la inversión extranjera y privatizar las empresas paraestatales. ${ }^{3}$ El Plan Baker fracasó por la falta de apoyo de la banca comercial y la oposición de gobiernos como los de Brasil y Perú. El Plan Brady substituyó al anterior en 1989 añadiendo algunas medidas para el alivio de la deuda y el servicio de la misma como los swaps de deuda-por-equidad puestos en práctica

1 Hills (en prensa), señala la presencia de ideólogos promotores de privatización en el Banco Mundial desde los años sesenta. Es preciso recordar que, si bien los regímenes de Salinas, Ménem y Pérez fueron precedidos por el Reaganismo y el Tatcherismo, éstos fueron a su vez precedidos por Paul Volker en la Reserva Federal de Estados Unidos en 1978, iniciando el desmantelamiento del fordismo global.

2 La crisis de la deuda externa de los años ochenta es, en realidad, la cuarta en la región. Las crisis anteriores ocurrieron en los años cercanos a 1820, 1870 y 1930.

3 El Plan Baker requería que la banca comercial y los países industrializados otorgaran créditos por 700,000 millones y 300,000 millones de dólares al año, respectivamente. 
desde 1985 por Chile y sus acreedores. ${ }^{4}$ México fue el primer país en renegociar la deuda bajo el nuevo esquema incluyendo la continuidad de swaps tras haberlos suspendido en 1987, siguiéndole Venezuela y Costa Rica. La relación entre deuda-renegociación-privatización de telecomunicaciones es un patrón que se repite en los países latinoamericanos (Figura 6). Aunque no en todos los casos se utiliza el mecanismo de swaps en la operación de venta, en el caso mexicano fue utilizado directamente (Grosse, 1992). ${ }^{5}$

Figura 6. Cronograma de privatización de compañías telefónicas

\begin{tabular}{|c|c|c|c|c|c|c|c|}
\hline & 1985 & 1986 & 1987 & 1988 & 1989 & 1990 & \\
\hline Argentina & & & swaps & & $\begin{array}{c}\text { Ménem } \\
\text { Plan }\end{array}$ & venta & \\
\hline${ }^{*}$ Chile & swaps & & Plan & venta & $\therefore$ & reventa & \\
\hline México & & swaps & propuesta & Salinas & GATT & venta & bolsa \\
\hline Venezuela & & & swaps & & $\begin{array}{l}\text { Pérez } \\
\text { GATT }\end{array}$ & & venta \\
\hline $\begin{array}{r}\text { Estados } \\
\text { Unidos }\end{array}$ & $\begin{array}{l}\text { Plan } \\
\text { Baker }\end{array}$ & & & & $\begin{array}{l}\text { Plan } \\
\text { Brady }\end{array}$ & & \\
\hline
\end{tabular}

El 9 de diciembre de 1990 el Secretario de Hacienda y Crédito Público anunció que el $20.4 \%$ del capital social de Telmex se adjudicaba al bloque integrado por el Grupo Carso (encabezado por Carlos Slim), France Cable and Radio (filial de France Telecomm), y Southwestern Bell en 1,757.6 millones de dólares. Con ello

4 Un swap consiste en la venta de documentos de deuda por las instituciones acreedoras a un precio inferior al nominal. Los inversionistas, generalmente compañías trasnacionales, negocian con el banco central del país deudor el canje del documento por una inversión en equidad a un valor igual o inferior al nominal. El primer swap en la historia se realizó en 1885 , cuando el gobierno de Costa Rica cedió acciones en sus ferrocarriles a cambio de deuda.

5 Tandon (1992) agrega que ocho meses antes de la venta, Telmex adquirió 670 millones de dólares de bonos del gobierno mexicano de un banco internacional por 291.4 millones de dólares. 
se ponía fin a 18 años de una telefonía operada por el Estado y 32 de estar en manos nacionales. Se ponía fin también a una subasta donde llegaron a la etapa final sólo otros dos grupos integrados por Acciones y Valores de México, ${ }^{6}$ GTE Telephone Corporation y Telefónica de España, ${ }^{7}$ cuya postura fue inferior en un $4 \%$ a la del ganador y el Grupo Gentor sin componente extranjero. En el camino había quedado la Casa de Bolsa Inverlat, así como una larga lista de compañías extranjeras: Nynex International, Bell Canada, Nippon Telephone and Telegraph (NTT), Cable and Wireless, Singapore Telecomm, United Telecommunications, U. S. Sprint y el Citibank. Todos los anteriores presentaron posturas formales a partir del anuncio de la desincorporación de Telmex hecho por el propio Presidente de la República en una asamblea general del Sindicato de Telefonistas de la República Mexicana el 18 de septiembre de 1989, mismo mes en el que se concluyó la renegociación de la deuda bajo el Plan Brady.

La decisión de Salinas fue el desenlace de una especie de danza en la cual por espacio de más de dos años diferentes funcionarios parecían turnarse para desmentir la posible venta de la compañía. Las negativas que alternadamente hicieron el titular de la SCT, tres sucesivos directores generales de la empresa, miembros del gabinete económico, el Secretario General del sindicato y el propio Presidente de la República fueron para apagar el rumor de que Telmex sería añadido a la lista de empresas paraestatales que fueron desincorporadas durante lagestión de Salinas como titular de la SPP. Dichas aseveraciones se originaron en la VIII Reunión Corporativa de Planeación en diciembre de 1987, cuando Carlos Casasús, Sub-director de Planeación Estratégica, presentó dicha idea en la ponencia "El cambio estructural en Telmex". En mayo de 1988 el entonces candidato Salinas se encargó de dar fuerza a los rumores cuando presidió el foro "Retos a la Modernización: Modernización del Sector Industrial, Infraestructura Nacional, Telecomunicaciones e Informática" en Monterrey. En dicho foro, empresarios regiomontanos propusieron la reprivatización de Telmex, a lo que el candidato respondió que existía un serio y preocupante retraso en los servicios telefónicos y expresó que en lo general coincidía con las ponencias.

6 Esta operadora de bolsa pertenece a Roberto Hernández, el mismo que se adjudicó Banamex, el banco más grande de México, sólo unas semanas después de haber perdido Telmex

7 Telefónica de España ya participa del mercado latinoamericano en Argentina, Chile, Venezuela y Puerto Rico, e intenta entrar a Paraguay y Uruguay. 
Aunado a lo anterior debe recordarse la campaña en los medios masivos orquestada contra Telmex, entre los que sobresalieron Televisa y los diarios Novedades y El Norte. Dicha campaña fue tan efectiva en insistir en la ineficiencia de Telmex que publicaciones como The New York Times y Telephony hicieron eco de ella en el extranjero. Coincidentemente detrás de la televisora privada y el diario capitalino figuraba prominentemente Rómulo O'farril, quien era uno de los principales accionistas de la compañía antes de convertirse en paraestatal en 1972 y es uno de los flamantes socios de Carlos Slim. Caso único ęn la historia de la publicidad oficial en México fue la contribución del propio Telmex mediante desplegados que anunciaban que la empresa no había alcanzado metas de instalación, operación y mantenimiento. Esta campaña de desprestigio jamás hizo alusión al hecho de que la empresa era sumamente rentable, alcanzando márgenes de utilidades de $40 \%$ y siendo la compañía que mejor cotizaba en la Bolsa Mexicana de Valores (Figuras 7 y 8 ).

Figura 7. Ingresos y Utilidades de Telmex 1982-1989

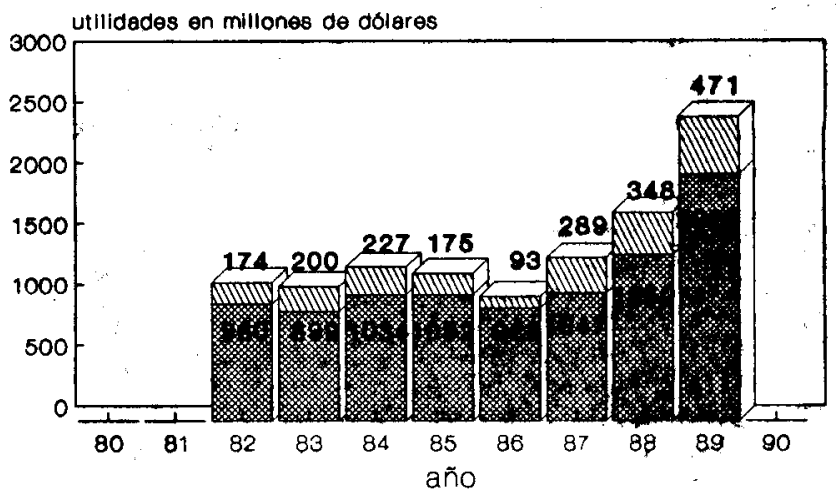

Mlli Ganancia nete

Fuentes: Pérez de Mendoza (1989: 95) de 1982-87; Teléfonos de México (1990a: $60)$ de 1988 y 89. 
Figura 8. Compañias líderes en la Bolsa Méxicana de Valores ${ }^{8}$

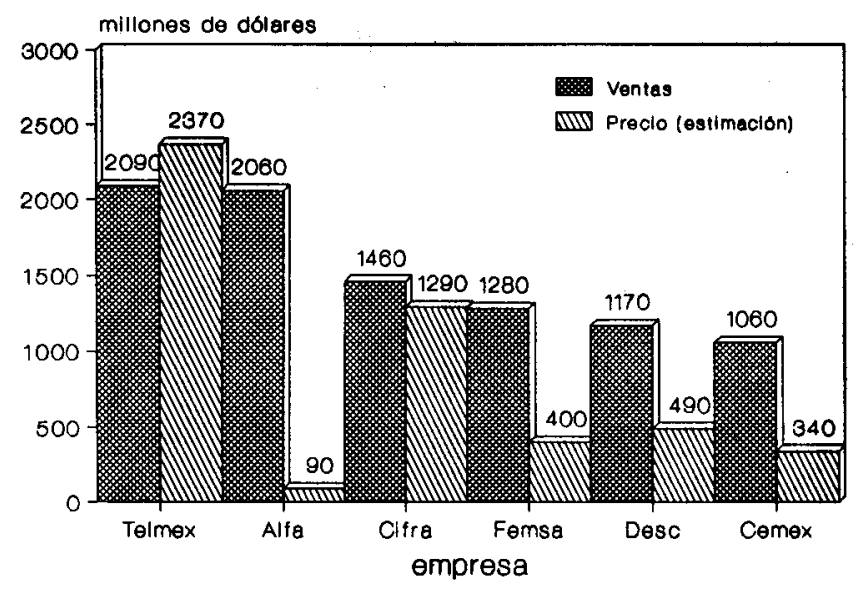

Elaborado con base en datos de Goldman (1990: 51).

Slim estaba considerado como el empresario número 54 de México con intereses en más de 43 empresas entre las que destacan Inversora Bursátil (Inbursa), Sanborn's, Frisco, Cigarros La Tabacalera, Compañía Hulera Euzkadi, Fábricas de Papel Loreto y Peña Pobre y Segumex. Gracias a Telmex, Slim ha escalado 52 posiciones en el ranking nacional y es ahora el segundo magnate sólo detrás de Emilio Azcárraga. No sorprende el hecho de no haber recurrido a capitales no especulativos y más establecidos como se hizo en Argentina con "Goyo" Pérez Companc o a compradores "naturales" en el sector como Televisa, cuya participación fue vetada. Esto se explica por el hecho de que Salinas ha utilizado la desincorporación del sector paraestatal para reajustar la correlación de fuerzas económicas, creando una nueva generación de capitalistas y limitando el creci-

8 Compañías con ventas superiores a los 1,000 millones de dólares para 1989. Las apreciaciones son para el periodo de junio de 1988 a mayo de 1989. 
miento doméstico de los viejos capitales. El caso de Televisa es claro ya que no sólo no se le permitió presentar postura para la adquisición de Telmex y se le rechazó la propuesta para que los satélites Solidaridad tuvieran transpondedores para DBS, sino que se alentó el crecimiento del Grupo Vargas otorgándole concesiones sin previo concurso y anunciando la desincorporación de las dos televisoras estatales en un paquete que incluye el diario El Nacional y la Compañía Operadora de Teatros (CCTSA). Después de un sexenio de haber crecido sólo en el extranjero, Televisa recibió finalmente la concesión de varias decenas de televisoras locales a unos días de hacer una contribución multimillonaria para la campaña presidencial del partido oficial que dará inicio en los próximos meses.

La misma prominencia de Carlos Slim en Telmex es engañosa, ya que aunque fue el principal comprador en la operación de Diciembre de 1990 y frecuentemente se cita su adquisición del $28.2 \%$ de las acciones AA, la mayoría de las acciones de Telmex no está en manos mexicanas. La compra del $49 \%$ de dichas acciones por parte de las dos compañías extranjeras y su posterior compra de acciones $A$ y $L$ aunadas a la venta de nuevos paquetes de acciones en Mayo de 1991 y 1992 hacen que la inversión extranjera ascienda a un total del 55.7\% (Figura 9). Más de un

Figura 9. Distribución de propiedad de compañías telefónicas

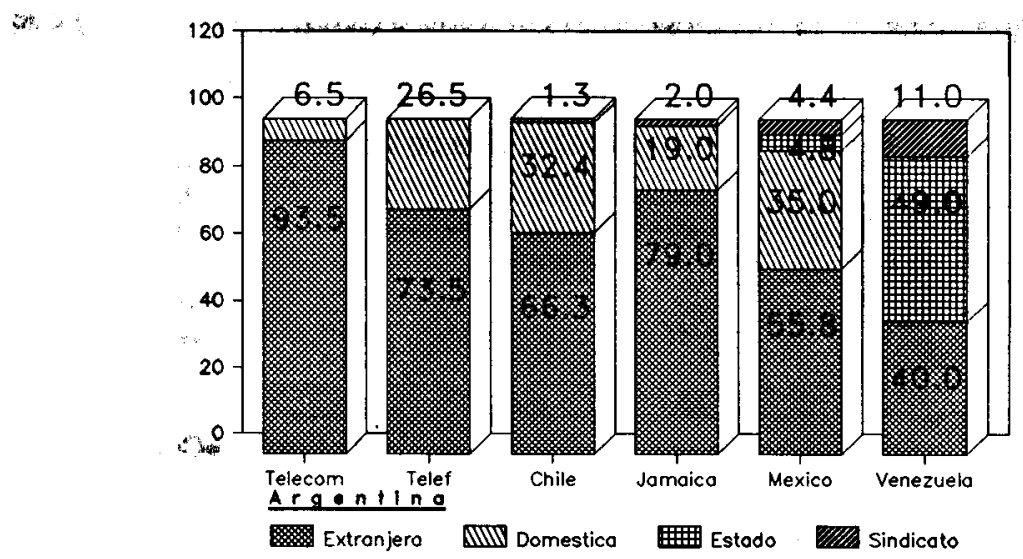

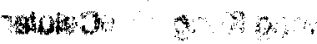


siglo después de que la ITT inició la industria telefónica en México seguida por L.M. Ericsson en 1907, el círculo se ha cerrado y según estimaciones del propio Banco Mundial, el $90 \%$ de las utilidades fluyen hacia afuera del territorio nacional (Tandon, 1992).

\section{Las baby bells en México y el mundo}

A pesar de que las compañías telefónicas regionales estadounidenses (baby bells) tienen menos de una década de haberse independizado de AT\&T ( $M a$ Bell) tienen una gran presencia internacional (Figura 10). En México, Southwestern Bell tiene gran parte de las acciones de Telmex, operación en la que quedó en el camino como postora su hermana Nynex. Bell South ha entrado al mercado celular en México y seis países más. Estas operaciones son sólo una mínima parte de las que en conjunto tienen las baby bells, las cuales han buscado los mercados internacionales a raíz de las severas limitaciones domésticas con las que nacieron en 1984 no permitiéndoles competir en la manufactura de equipos, servicios de larga distancia y servicios de información, además de estarles vedado el mercado de televisión por cable y tener un margen de utilidades máximo de 15\%. Dichas restricciones parecen haberlas beneficiado en el largo plazo, pues no sólo tienen operaciones con un mayor margen de utilidades en el extranjero, sino que han pasado por procesos de integración vertical y horizontal que las han vuelto competitivas internacionalmente y se encuentran listas a capturar mercados domésticos (televisión por cable) que les habían sido vedados.

Figura 10. Inversiones internacionales de las baby bells

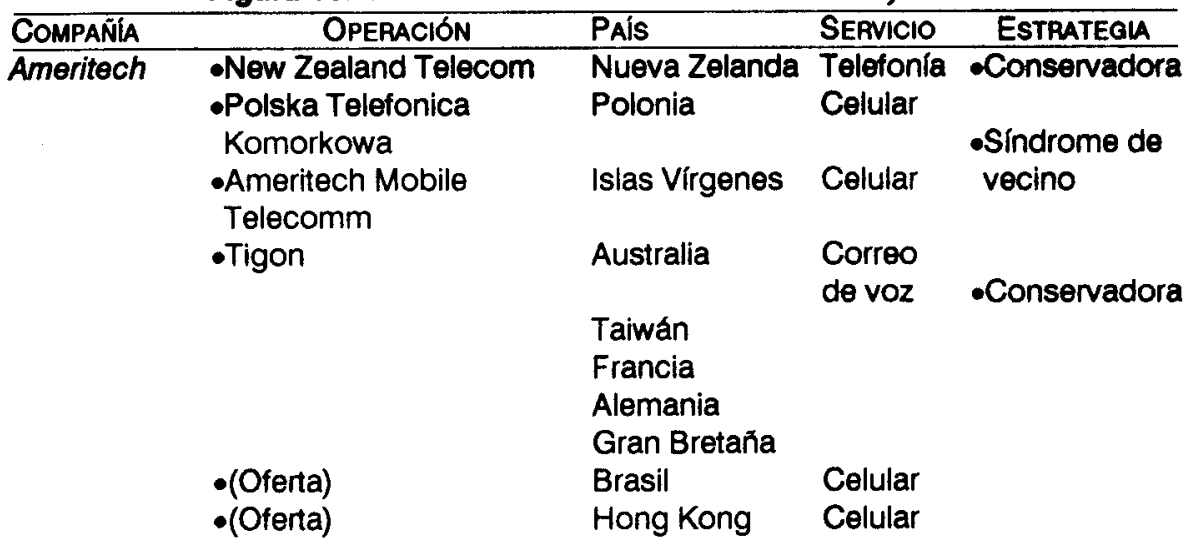


Figura 10 (continuación). Inversiones internacionales de las baby bells

\begin{tabular}{|c|c|c|c|c|}
\hline \multirow[t]{3}{*}{$\begin{array}{l}\text { Compañía } \\
\text { Bell Atlantic }\end{array}$} & $\begin{array}{l}\text { OPERACIÓN } \\
\text { - New Zealand Telecom } \\
\text {-Eurotel }\end{array}$ & $\begin{array}{l}\text { PAís } \\
\text { Nueva Zelanda } \\
\text { Checoslovaquia }\end{array}$ & $\begin{array}{l}\text { Servicio } \\
\text { Telefonía } \\
\text { Celular }\end{array}$ & $\begin{array}{l}\text { Estrategia } \\
\text {-A largo plazo } \\
\text { - Merc. Desreg. }\end{array}$ \\
\hline & $\bullet N D$ & CEI & Celular & \\
\hline & $\begin{array}{l}\text {-Sorbus } \\
\text {-(Oferta) }\end{array}$ & $\begin{array}{l}\text { Gran Bretaña } \\
\text { Brasil }\end{array}$ & \multicolumn{2}{|c|}{$\begin{array}{l}\text { Informática } \\
\text { Celular }\end{array}$} \\
\hline \multirow[t]{8}{*}{ Bell South } & $\begin{array}{l}\text {-China Bell South Int. } \\
\text {-Cía. de Radiocom. Moville }\end{array}$ & $\begin{array}{l}\text { China } \\
\text { eArgentina }\end{array}$ & $\begin{array}{l}\text { Telefonia } \\
\text { Celular }\end{array}$ & \multirow[t]{8}{*}{$\begin{array}{l}\text { - Merc. grandes } \\
\text { - Celular }\end{array}$} \\
\hline & •Cidcom & Chile & Celular & \\
\hline & -Comcel & México & Celular & \\
\hline & -Abiatar & Uruguay & Celular & \\
\hline & •TelCel & Venezuela & Celular & \\
\hline & •ND & Francia & Celular & \\
\hline & $\bullet N D$ & Alemania & Celular & \\
\hline & •(Oferta) & Gran Bretaña & Celular & \\
\hline Nynex & - Gibraltar Telephone Co. & España & Telefonía & -Serv. inalámb. \\
\hline \multirow{5}{*}{$\begin{array}{l}\text { Pacific } \\
\text { Telesis }\end{array}$} & •Cidcom & Chile & Celular & \multirow[t]{5}{*}{-Conservadora } \\
\hline & •ND & Gran Bretaña & Celular & \\
\hline & $\bullet N D$ & Gran Bretaña & Cable TV & \\
\hline & -ND & Corea del Sur & $\begin{array}{l}\text { Servicios } \\
\text { de va }\end{array}$ & \\
\hline & -(Oferta) & Corea del Sur & Celular & \\
\hline \multicolumn{2}{|c|}{$\begin{array}{l}\text { Southwestern } \bullet \text { Telmex } \\
\text { Bell }\end{array}$} & México & Telefonía & \multirow{2}{*}{$\begin{array}{l}\text { - A largo plazo } \\
\text { - Síndrome de } \\
\text { vecino }\end{array}$} \\
\hline & •ND & Gran Bretaña & Cable & \\
\hline \multirow[t]{6}{*}{ us West } & $\begin{array}{l}\text {-Central Europe Fiber } \\
\text { Optica System }\end{array}$ & $\begin{array}{l}\text { Bulgaria } \\
\text { Checoslovaquia } \\
\text { Hungria } \\
\text { Israel } \\
\text { Polonia } \\
\text { Rumania } \\
\text { Turquía }\end{array}$ & Telefonía & \multirow[t]{6}{*}{ Soc. Minorit. } \\
\hline & •Eurotel & Checoslovaquia & Celular & \\
\hline & -Westel Radiotelfon & Hungría & Celular & \\
\hline & $\bullet N D$ & CEI & Telefonía & \\
\hline & $\bullet N D$ & Gran Bretaña & Cable & \\
\hline & •ND & Francia & Cable & \\
\hline
\end{tabular}

Elaborado con datos de Sims (1989) y Bagchi-Sen y Parthavi Das (en prensa). 
Las baby bells tienen ahora la experiencia para competir o cartelizarse en los mercados que se abren como el mexicano. El título de concesión reserva a Telmex los servicios de larga distancia por el resto del siglo y el servicio básico por varias décadas más y el TLC abre a la inversión extranjera servicios como MDS y videotexto. Sin embargo, los negociadores mexicanos pagan su falta de experiencia y visión al ignorar la creciente convergencia e hibridación de tecnologías donde su diferencia es cada vez más irrelevante. Mientras el equipo negociador mexicano asegura que la diferencia entre servicios como la telefonía básica y los de valor agregado es perfectamente clara, denotan que no han aprendido ni siquiera las lecciones sufridas en carne propia como la telefonía celular que estuvo a punto de ser monopolizada por Alejo Peralta debido a la falta de conocimientos acerca la tecnología por parte de los órganos responsables. El gobierno federal difícilmente podrá concertar de la misma manera con compañías como Call-Net que fue apoyada por el gobierno canadiense para que se consideraran como de valor agregado servicios que ya son comunes como Call Accounting y Call Identification (Mosco, 1990).

\section{Consideraciones finales}

La inversión extranjera en servicios de telecomunicación en América Latina ha crecido vertiginosamente y lo seguirá haciendo dadas las condiciones en el futuro cercano. La visión optimista de Reich sobre la irrelevancia de la nacionalidad del capital sólo es posible porque detrás se encuentran visiones como la de Gore que perpetúa el subsidio y protección del sector privado. La "supercarretera electrónica" es sólo el más nuevo "instrumento escogido" para que la mano visible del Estado "estimule" al sector privado en la competencia internacional como se hizo con la RCA en el radio, la Comsat con satélites, MCC y Sematech con los superconductores y los consorcios de HDTV. El gobierno de Japón ha hecho lo suyo con la construcción de una red digital para unir 15 Technopolis orquestada por el MITI y con la participación de las grandes firmas niponas de telecomunicaciones. Mientras tanto el Estado mexicano toma como artículo de fe la globalización mano invisible y sólo se permite tener un rol de inversionista en infraestructura. Después de todo, la mejor garantía para la inversión extranjera es que la tasa de amortización sea inferior a la del Estado anfitrión (Gordon, 1988). 
Ejercicios como el presente corren el riesgo de ser leídos como propuestas nacionalistas. El hecho de que se analicen críticamente los discursos sobre globalización no implica alinearse con el esquema que es reemplazado. Es preciso construir discursos articulen los análisis acerca de los diversos ejes de dominación como clase, género y etnia para evitar la trampa del falso debate entre los polos globalización versus nacionalismo.

\section{Referencias bibliográficas}

BARRERA Eduardo (s.f.): "The privatization of telecommunications in Latin América: The role of domestic capital". En Bella MODDY, Joseph STRAUBHAAR, Johanes BAUER y David MCCARTHY (eds.): The Privatzation of Telecommunications in the Third World and Eastern Europe. (en prensa).

BAGCHI-SEN Shartmistha y Parthavi DAS (s.f.): "Foreign Direct Investment by U. S. Telecommunication Companies". En Bella MOdDY, Joseph STRAubHAAR, Johanes BAUER y David MCCARTHY (eds.): The Privatzation of Telecommunications in the Third World and Eastern Europe. (en prensa).

Business Week (1993): “1-800-GUTS”. Núm. 3334, pp. 26-32 (30 de agosto).

CAREY James (1989): Communication as Culture: Essays on Media and Society. Boston: Unwin Hyman.

CleAver Harry (1990): "Close the IMF, abolish debt and end development: A class analysis of the international debt crisis". Capital and Class, pp. 17-50.

COCKCROFT James D.(1979): El imperialismo, la lucha de clases y el Estado en México. Mexico City: Editorial Nuestro Tiempo.

GolDMAN David (1990): “A revolution you can invest in”. Forbes, pp. $48-54$ (9 de julio).

GordoN David M. (1988): "The Global Economy: New Edifice or Crumbling Foundations?". New Left Review Núm. 168, pp. 24-65 (marzo-abril). 
GROSSERobert (1992): Private Sector Solutions tothe Latin American Debt Problem. New Brunswick: Transaction Publishers.

HILLS Jill (s.f.) "The ideology of privatization and the World Bank". En Bella Moddy, Joseph STRAUbHAAR, Johanes BAUER y David MCCARTHY (eds.): The Privatzation of Telecommunications in the Third World and Eastem Europe. (en prensa).

MATTELART Armand (1991): Advertising International: The Privatisation of Public Space. Nueva York: Routledge.

MÉNDEZ MORENo Rafael (1967): El telégrafo en el destino nacional . México, D.F.: Imprenta Arana.

Mosco Vincent (1989): The Pay-per Society: Computers and Communication in the Information Age. Norwood, NJ: Ablex.

(1990): "Toward a Transnational World Information Order: The CanadaU. S. Free Trade Agreement", Canadian Joumal of Communication vol. 15, Núm. 2, pp.46-63 (May).

PÉrez Mendoza Antonio (1989): "Teléfonos de México: Development and Perspectives". En Peter F. CoWHEY, Jonathan D. ARONSON y Gabriel SZEKELY (eds.): Changing Networks: Mexico's Telecommunications Options. La Jolla, Ca: Center for U. S. - Mexican Studies, UCSD, pp. 91-99.

REICH Robert B. (1991): The Work of Nations. Nueva York: Alfred A. Knopf.

RUTTEN Paul (1991): "Local Popular Music on the National and International Markets". Cultural Studies Vol. 5, Núm. 3, pp. 284 - 295 (octubre).

SAMARAJIVA Rohan (1990): "Towards a Theoretical Understanding of Telecommunication Policy in the Third World". Ponencia presentada en el $17^{\circ}$ Congreso de AIERI. Bled, Yugoslavia. 
SÁNCHEZ Jesús (1987): “La coordinación de comunicaciones, punta de lanza del esfuerzo modernizador: Salinas”. El Financiero, 15 de octubre.

SCHILLER Herbert (1992): Mass Communications and American Empire. Boulder: Westview Press. $2^{\text {a }}$ edición.

Sims Calvin (1989): "The Baby Bells Scramble for Europe". The New York Times, 10 de diciembre, p. 1, sección 3.

SMITH Anthony (1991): The Age of Behemoths: The Globalization of Mass Media Firms. Nueva York: Priority Press Publications.

SMITH Ralph Lee (1970): "The Wired Nation". The Atlantic (s.d.).

TANDOM Pankaj (1992): "México. Background, Telmex, Aeroméxico, Mexicana". Documento presentado en la World Bank Conference on the Welfare Consequences of Selling Public Enterprises, 11 y 12 de junio, Washington, D. C.

TAPIO Varis (1984): “The Internatio Flow of Television Programs". Joumal of Communication Vol. 24, Núm. 1, pp. 143 - 152.

Teléfonos de México, S. A. de C. V. (1990): Informe Anual 1989. México, D. F.: Telmex. 